\title{
Consensus guidelines for the diagnosis and treatment of adults with GH deficiency II: a statement of the GH Research Society in association with the European Society for Pediatric Endocrinology, Lawson Wilkins Society, European Society of Endocrinology, Japan Endocrine Society, and Endocrine Society of Australia
}

\author{
Ken K Y Ho on behalf of the 2007 GH Deficiency Consensus Workshop Participants \\ Pituitary Research Unit, Garvan Institute of Medical Research, 384 Victoria Street, Darlinghurst, Sydney, NSW 2010, Australia \\ (Correspondence should be addressed to K KY Ho; Email: k.ho@garvan.org.au)
}

\begin{abstract}
Objective: The GH Research Society held a Consensus Workshop in Sydney, Australia, 2007 to incorporate the important advances in the management of GH deficiency (GHD) in adults, which have taken place since the inaugural 1997 Consensus Workshop.

Method: Two commissioned review papers, previously published Consensus Statements of the Society and key questions were circulated before the Workshop, which comprised a rigorous structure of review with breakout discussion groups. A writing group transcribed the summary group reports for drafting in a plenary forum on the last day. All participants were sent a polished draft for additional comments and gave signed approval to the final revision.

Conclusion: Testing for GHD should be extended from hypothalamic-pituitary disease and cranial irradiation to include traumatic brain injury. Testing may indicate isolated GHD; however, idiopathic isolated GHD occurring de novo in the adult is not a recognized entity. The insulin tolerance test, combined administration of GHRH with arginine or growth hormone-releasing peptide, and glucagon are validated GH stimulation tests in the adult. A low IGF-I is a reliable diagnostic indicator of GHD in the presence of hypopituitarism, but a normal IGF-I does not rule out GHD. GH status should be reevaluated in the transition age for continued treatment to complete somatic development. Interaction of GH with other axes may influence thyroid, glucocorticoid, and sex hormone requirements. Response should be assessed clinically by monitoring biochemistry, body composition, and quality of life. There is no evidence that GH replacement increases the risk of tumor recurrence or de novo malignancy.
\end{abstract}

European Journal of Endocrinology 157 695-700

\section{Introduction}

Growth hormone deficiency (GHD) is a well-recognized clinical entity in the adult. It causes abnormalities in substrate metabolism, body composition, physical, and psychosocial function, which improve with GH replacement. In April 1997, the GH Research Society (GRS) convened an international workshop that formulated Consensus Guidelines for the Diagnosis and Treatment of Adults with GHD, which were widely adopted internationally by the health authorities and professional societies (1). The GRS convened a second international workshop on March 13-15, 2007 in Sydney, Australia, to review and incorporate important advances that have occurred in the last 10 years. The GRS invited leading experts in the field and related professional societies, all of whom contributed to the updated Consensus.

The 2007 Workshop was structured in terms of format and discussions using the 1997 document as a template and produced this comprehensive statement that integrates new recommendations to those generated in 1997. The reader is encouraged to read the first document for further details (1).

Two discussion documents were commissioned for the workshop: one on the diagnosis and the other on the therapy of adults with GHD. These two review papers will be published separately $(2,3)$. Two previously published Consensus Statements for the GRS, the initial 1997 document (1) and another on the management of the GH-treated adolescent in the transition to adult care (4), along with the two commissioned reviews were 
circulated before the Workshop. These collective papers provided the background material on which the workshop discussions were based. A set of questions addressing unresolved issues in the diagnosis and management was circulated to all participants. Review lectures and focused presentations of relevant topics were given by invited experts. A writing group transcribed the summary group reports for consensus drafting in a plenary forum on the last day. Participants signed an agreement form at the end of the workshop. They were sent a polished draft for additional comments and gave signed approval to the final revision.

The workshop was attended by 30 delegates including authorities in the field and representatives from related professional societies involved in the care of pediatric and adult patients with GHD. The meeting was supported by the GRS and unrestricted grants from its corporate members.

\section{Diagnosis of GHD in adulthood}

Severe GHD is defined biochemically within an appropriate clinical context. In patients with hypothalamic-pituitary disease, the syndrome of adult GHD characteristically manifests with derangements in body composition, physical, and psychological function.

\section{Who to test}

The current recommendations extend beyond those of 1997 and state that patients who should be tested for GHD are those who show evidence of hypothalamicpituitary disease, and in whom there is an intention to treat. This includes patients from the following three groups:

1) Those with signs and symptoms of hypothalamicpituitary disease (endocrine, structural, and/or genetic causes)

2) Those who have received cranial irradiation or tumor treatment

3) Those with traumatic brain injury (TBI) or subarachnoid hemorrhage

TBI is now appreciated as a cause of hypopituitarism. The severity of TBI is not well correlated to the degree of pituitary dysfunction. As the GH axis may recover after TBI, testing for GHD should be undertaken no sooner than 12 months after the injury.

It is recognized that GH sufficiency to deficiency is a continuum. The biochemical diagnosis of severe GHD is straightforward. Partial GHD is at present not a welldefined clinical entity in adults. The presence of a low serum insulin-like growth factor-I (IGF-I) concentration increases the likelihood of GHD. Inconclusive testing should be followed by ongoing clinical evaluation and repeat testing.
Testing may indicate isolated GHD; however, idiopathic isolated GHD occurring de novo in the adult is not a recognized diagnostic entity; therefore testing without evidence of the patient falling into one of the above groups should not be undertaken. This is in contrast to the patient with objective evidence of hypothalamicpituitary disease (e.g., on imaging or after irradiation), who may present with organic isolated GHD as the first hormonal deficiency, and this may account for up to $25 \%$ of cases of GHD in the adult.

\section{Diagnostic tests for GHD}

Historically, many stimulation tests have been used. The 1997 Workshop recommended the insulin tolerance test (ITT) to be the diagnostic test of choice, with the combined administration of arginine and GH-releasing hormone (GHRH) as the most promising alternative. Administration of arginine alone or glucagon could be considered, but had less established diagnostic value.

\section{Stimulation tests}

Based on a review of work since then, the 2007 Workshop agreed that GHRH+arginine, GHRH+ growth hormone-releasing peptide (GHRP), and glucagon stimulation tests are also now well validated in adults. The ITT evaluates the integrity of the hypothalamic-pituitary axis and has the added advantage of also stimulating adrenocorticotrophin, while the combined tests evaluate maximal secretory capacity. The glucagon test is a suitable alternative when ITT is contraindicated or when GHRH or GHRP are not available. Ghrelin mimetics alone are currently under evaluation as a test of the $\mathrm{GH}$ axis.

One stimulation test is sufficient for the diagnosis of adult GHD. Not all patients suspected of having GHD, however, require a GH stimulation test for diagnosis. Patients with three or more pituitary hormone deficiencies and an IGF-I level below the reference range have $>97 \%$ chance of being GHD, and therefore do not need a $\mathrm{GH}$ stimulation test.

Each test has limitations; the ITT can be contraindicated in patients with ischemic heart disease or seizures, and in the elderly. The responses to all tests show intraindividual variability. All of the tests are appropriate for the first two categories of patients mentioned above. However, since the combined tests stimulate both the hypothalamus and the pituitary, GHD due to hypothalamic disease may be missed. This is exemplified by the studies in those treated with cranial irradiation, in which the ITT shows the greatest sensitivity and specificity within the first 5 years after irradiation. If the peak GH level during a GHRH+ arginine test is normal in those who have received irradiation, then an ITT should also be performed. In irradiated patients as well as those with inflammatory and infiltrative lesions, GHD may develop many years 
after the initial insult. Therefore, this group should be followed in the long term with repeat testing as clinically indicated.

The cutoff for the diagnosis of GHD varies with the test used. For the ITT and glucagons test, the validated cutoff for GHD in adults is a peak GH response of $<3 \mu \mathrm{g} / \mathrm{l}$. The following cutoff levels have been validated for GHRH + arginine: for those with a body mass index (BMI) $<25 \mathrm{~kg} / \mathrm{m}^{2}$, a peak $\mathrm{GH}<11 \mu \mathrm{g} / \mathrm{l}$; for BMI $25-30 \mathrm{~kg} / \mathrm{m}^{2}$, a peak $\mathrm{GH}<8 \mu \mathrm{g} / \mathrm{l}$; for BMI $>30 \mathrm{~kg} / \mathrm{m}^{2}$, a peak $\mathrm{GH}<4 \mu \mathrm{g} / \mathrm{l}$. Clonidine, L-DOPA, and arginine are not useful tests in adults. The arginine test alone is used in the transition period, but the response is very dependent on BMI; therefore the arginine test should be limited to non-obese adolescents. A limitation for most tests is the relative lack of validated normative data based on age, gender, and BMI. These tests should be performed in experienced endocrine units where such tests are performed frequently.

\section{Biochemical markers of GH action}

IGF-I is a good screening test for GHD in younger lean patients $\left(<40\right.$ years, BMI $\left.<25 \mathrm{~kg} / \mathrm{m}^{2}\right)$ with evidence of hypopituitarism; however, a normal IGF-I does not rule out GHD at any age. The levels of IGF-I depend on many factors other than GH status. For example, in obesity the GH secretion is suppressed, but IGF-I is normal. In undernourished patients, IGF-I levels are low. Currently, there are no alternative markers of $\mathrm{GH}$ action, which are superior to IGF-I. The 2007 Statement reaffirms the value of IGF-I in the evaluation of the adult suspected of having GHD.

\section{Transition age patients}

The 1997 Consensus did not include an evaluation of this group of patients, for whom strong evidence now exists that full somatic maturation is not achieved if $\mathrm{GH}$ is not continued after cessation of growth.

In patients with childhood-onset GHD, the need for continuation of $\mathrm{GH}$ replacement should be evaluated following completion of statural growth (usually before the age of 20 years). GH testing is not required for those with a transcription factor mutation (e.g., POU1F1 (Pit-1), PROP-1, HESX-1, LHX-3, LHX-4), those with more than three pituitary hormone deficits, and those with isolated GHD associated with an identified mutation (e.g., GH-1, GHRH-R). All other patients should undergo GH testing after at least 1 month off GH treatment.

For non-GHD pediatric indications (e.g., Turners syndrome, small for gestational age), there is no proved benefit of GH treatment in the adult; therefore there is no indication to evaluate $\mathrm{GH}$ status at the completion of growth.

For the ITT, the suggested cutoff in the transition period, based on present evidence, is a GH peak $<6 \mu \mathrm{g} / \mathrm{l}$; however, further validation is required. A second re-investigation at the completion of somatic growth (approximate age 25 years) can be considered in those with childhood-onset isolated idiopathic GHD before a commitment is made to lifelong GH replacement. Reevaluation at this time may also be justified for patients who had discordant tests at the transition age (normal GH on stimulation but low IGF-I) and were not treated with $\mathrm{GH}$.

\section{Assays for GH and IGF-I}

The problem of the lack of assay standardization has not been addressed since the 1997 workshop when the issue was highlighted. The analytical method influences the results of GH stimulation tests, and ideally assay-specific cutoff values should be defined for each stimulation test. Substantial heterogeneity exists among currently utilized assays, which would be reduced by the adoption of a universal GH calibration standard. The GRS advocates the use of recombinant $22 \mathrm{kDa} \mathrm{GH}$ calibrator (International Reference Preparation (IRP) 98/574) in all GH assays.

The GRS calls for assay manufacturers to publish the validation of their assay, which should include specification of the GH isoforms detected (20 kDa GH, $22 \mathrm{kDa}$ $\mathrm{GH}$, and other isoforms) and the presence or absence of any effects due to $\mathrm{GH}$ binding protein.

Similar problems exist for measuring IGF-I. The technology exists for the accurate measurement of IGF-I. A universal calibrator is essential and should be recombinant human IGF-I of the highest purity. This should be adopted by all assay manufacturers. There is an urgent need for normative data. The utility of IGF-I measurements will be enhanced by specifying age and gender normal ranges. For example, a reference range has recently been established for the Japanese population from a dataset of over 1000 individuals.

\section{Treatment of GHD in adults}

The goal of replacement therapy is to correct the metabolic, functional, and psychological abnormalities associated with adult GHD. The Workshop reaffirmed the 1997 Statement that all patients with documented severe GHD are eligible for GH replacement, while incorporating new recommendations in the areas of dosing, hormone interactions, efficacy, safety, the care of the young adult after attainment of final height, and of the elderly with GHD.

\section{Dosing guidelines}

The objectives of treatment are to maximize benefit and minimize side effects. GH secretion is greater in younger individuals than older ones, and in women than men. Based upon this knowledge and a large amount of clinical experience, it is recommended that the starting dose of $\mathrm{GH}$ in young men and women be 0.2 and $0.3 \mathrm{mg} /$ day respectively, and in older individuals 
$0.1 \mathrm{mg} /$ day. Dose determination based on body weight is not recommended due to large interindividual variation in absorption, in sensitivity to $\mathrm{GH}$ and the lack of evidence that a larger replacement dose is required for heavier individuals in adults. It is recommended that $\mathrm{GH}$ be administered in the evening to mimic the greater secretion of $\mathrm{GH}$ at night. Dose escalation should be gradual, individualized, and guided by clinical and biochemical response (see below). Long-acting preparations of human $\mathrm{GH}$ are under evaluation for longterm safety and efficacy. The doses used in the adolescent in transition have typically been intermediate doses between the pediatric doses required during the growth years and the adult dose.

\section{Hormone interactions}

\section{Sex steroid therapy}

Sex steroid replacement should be optimized before GH testing or initiation of GH replacement therapy. Studies of the interactions between sex steroid replacement and $\mathrm{GH}$ action have shown that estrogen administered by the oral route impairs $\mathrm{GH}$ action, leading to higher dose requirements. It is preferable for estrogen in hypopituitary women to be replaced physiologically by a non-oral route, since the GH requirements will be reduced. Sex steroid replacement after the time of normal menopause should be based on current recommendations for the general population. Any modification in oral estrogen dose requires reevaluation of the $\mathrm{GH}$ dose. In contrast to estrogen, these considerations do not apply to androgen replacement therapy.

\section{Glucocorticoid replacement therapy}

GH and IGF-I influence glucocorticoid metabolism by regulating the activity of $11 \beta$-hydroxysteroid dehydrogenase, type 1 (11 $\beta$-HSD1), an enzyme converting inactive cortisone to cortisol. Initiation of $\mathrm{GH}$ replacement may unmask secondary adrenal insufficiency in some patients by reducing the activity of $11 \beta$-HSD1. In the patient with central adrenal failure, initiation of $\mathrm{GH}$ treatment may require an increase in hydrocortisone dose. Careful monitoring of patient's symptoms such as weight, appetite, and mood are required to assess the need for glucocorticoid dose modification.

\section{Thyroid function}

Thyrotrophin measurements are not helpful in the hypopituitary patient. As GH increases the peripheral conversion of tri-iodothyronine to thyroxine $\left(\mathrm{T}_{4}\right), \mathrm{GH}$ treatment may unmask preexisting central hypothyroidism, which is recognized by a fall of serum $\mathrm{T}_{4}$ into the subnormal range. In thyroxine-replaced patients, $\mathrm{GH}$ substitution may necessitate adjustment of the thyroid hormone dose.

\section{Monitoring efficacy}

A careful clinical examination should be undertaken with weight, height, and BMI recorded before commencing replacement therapy. Objective parameters such as body composition should be used to monitor the response to GH therapy. Body composition can be measured by simple anthropometry, such as waist circumference. Internationally accepted recommendations for waist circumference such as those defined by the National Cholesterol Education Program, Adult Treatment Panel III (NCEP ATP III) or International Diabetes Federation and where available ethnic-based guidelines should be used. Body composition assessment by anthropometric measurements should be performed at least yearly.

Where available, dual X-ray absorptiometry (DEXA) should be used to quantify changes in body composition. It gives an accurate measure of lean mass and fat mass. DEXA is also a reliable tool for assessing bone density, an important parameter that improves with $\mathrm{GH}$ replacement therapy. During the first year, bone mineral density may fall during $\mathrm{GH}$ treatment due to increased bone remodeling. Therefore, it is recommended that DEXA be performed at baseline and every 2 years thereafter. If treatment targets for bone density are not attained, a review of GH dose and additional medications should be undertaken.

Serum IGF-I is an indicator of hepatic GH action, and it is the most useful serum marker for GH dose titration in adults. It should be measured at least yearly. In the event of GH dose adjustment, assessment should be performed no sooner than 6 weeks after a dose change. IGF-I levels should be maintained below the age- and gender-related upper limit of normal, including in those patients with proved GHD, who present with normal IGF-I levels at baseline.

Hypopituitary patients are at increased risk for cardiovascular disease. No firm outcome data exist with regard to the effects of $\mathrm{GH}$ replacement therapy on cardiovascular events. A meta-analysis of placebocontrolled trials has indicated that an improvement of surrogate markers such as diastolic blood pressure, fat mass, total, and low density lipoprotein (LDL) cholesterol occurs with GH replacement therapy. Therefore, in addition to waist circumference measurements, these cardiovascular risk markers should be measured yearly in all patients. The cardiovascular treatment goals for the adult patient with GHD should be the same as for the general population, with all values maintained within the age- and gender-related normal range. Fasting glucose levels should be monitored yearly because of the increased prevalence of obesity in these patients.

Quality of life (QOL) in adult patients with GHD is impaired. A careful history with attention to energy level, partner satisfaction, sick days, and vitality is of value in monitoring treatment response. Disease-specific QOL questionnaires that assess the problems need to be 
validated for country, ethnicity, and language, and are usually reserved for research purposes.

\section{Treatment goals}

\section{GH treatment beyond attainment of adult height}

The beneficial effects of GH replacement therapy have now been convincingly demonstrated throughout the lifespan. The goal of treatment after cessation of linear growth is to achieve full somatic development including the accrual of maximal bone and muscle mass. GH treatment should be continued in all young adults with persistent GHD after attaining final height. GH-deficient adolescents who decline therapy should be closely monitored. Evidence of body composition abnormalities should be a strong indication to restart $\mathrm{GH}$, and such a finding should lead to a discussion with the patient regarding advantages of treatment. During the transition period, the pediatric endocrinologist should seek transition arrangements with adult endocrinologists for continuity of care.

\section{Adult onset GHD}

The goals of GH replacement therapy in adult onset GHD are improved body composition, preserved skeletal mass, normalized cardiovascular risk factors, maintenance of normal IGF-I status, and an optimal level of physical and psychological functioning.

\section{The elderly patient with GHD}

The age-related decline in the GH-IGF-I status does not warrant GH supplementation; however, patients with proved GHD should be treated. In the elderly GHD patient, treatment can be achieved with lower doses, concordant with the observed physiological decrease in GH secretion. The elderly are known to be more sensitive to $\mathrm{GH}$ and prone to side effects; therefore the dose should be adjusted carefully.

\section{Safety \\ Insulin resistance}

Increased knowledge of GH physiology has lead to dose adjustments that have substantially reduced the incidence of side effects, and GH is now recognized as a safe therapy when standards of care are followed. GH replacement therapy is not associated with an increased incidence of either type 1 or type 2 diabetes mellitus. It does, however, increase insulin resistance and may at times lead to worsening of glucose tolerance. Thus, individuals predisposed to type 2 diabetes, such as those with a positive family history, or who are obese or older, require careful monitoring. If type 2 diabetes is diagnosed, it should be managed similarly to any other patient with this disease, and GH replacement therapy continued.

\section{Hypothalamic/pituitary tumor recurrence}

There is no evidence that hypothalamic or pituitary tumor recurrence is influenced by $\mathrm{GH}$ replacement therapy. Before GH replacement therapy is initiated, pituitary imaging should be performed. Good clinical practice predicates that patients with residual tumors should be monitored regularly; GH replacement therapy does not impose a need for intensifying follow-up.

\section{Malignancy risk}

There is no evidence that GH replacement in adults increases the risk of de novo malignancy or recurrence. GH treatment during childhood of survivors of cancer treatment increases slightly the relative risk of a second neoplasia, but there are no comparable data in adults. GH therapy should be halted in any patient with active malignancy until the underlying condition is controlled. Because GH replacement therapy has not been associated with an increase in cancer risk, current recommendations for cancer prevention and early detection in the general population should be implemented.

\section{Conclusion}

Testing for GHD should be undertaken with an intention to treat in patients with hypothalamic-pituitary disease, those who have received cranial irradiation, and those with TBI or sub-arachnoid hemorrhage. The diagnosis of severe GHD is straightforward but partial GHD is not adequately defined. Testing may indicate isolated GHD; however, idiopathic isolated GHD occurring de novo in the adult is not a recognized entity. Insulin-induced hypoglycemia, combined administration of GHRH with arginine or GHRP, and glucagon are validated stimulatory tests for the diagnosis of GHD in the adult. A low IGF-I is a reliable diagnostic indicator of GHD in patients with hypopituitarism; however, a normal IGF-I does not rule out GHD. Assay standardization remains an important unresolved issue. Universally adopted calibrators for GH and IGF-I assays are required. The availability of age- and sex-specific normative data for IGF-I assays would be highly advantageous to clinical management. The benefits of GH replacement have been demonstrated throughout life. Thus, GH status should be reevaluated in the transition age for continued GH replacement to achieve full somatic development. Replacement therapy should be individualized based on the titration against the serum IGF-I levels and the absence of adverse events. Interaction of GH with other hormone axes may influence thyroid, glucocorticoid, and sex hormone requirements. Response should be evaluated by 
monitoring biochemistries (IGF-I, glucose, lipids), weight, and body composition. QOL assessment is important but does not necessarily require a questionnaire. There is no evidence that GH replacement increases the risk of tumor recurrence or de novo malignancy.

\section{Acknowledgements}

A Program Committee (Peter Clayton, Gudmundur Johannsson, and Ken Ho) formulated the Workshop program and was responsible for the final drafting of the Consensus Statement. The Consensus Workshop was organized by the GH Research Society and supported in part by unrestricted grants from Ferring, Genetech, JCR Pharmaceuticals, Eli Lilly, Novo Nordisk, Pfizer, and Serono. The participants at the Workshop were: Bert Bakker, Genentech, San Francisco, CA, USA, Vita Birzniece, Garvan Institute of Medical Research, Sydney, Australia, Felipe Casanueva, Department of Medicine Endocrine Division, Santiago de Compostela, Spain, Agnes Champigneulle, Merck Serono, Geneva, Switzerland, Kazuo Chihara, University of Kobe, Kobe, Japan, Jens Sandahl Christiansen, European Society of Endocrinology and Aarhus University Hospital, Aarhus, Denmark, Peter Clayton, European Society of Paediatric Endocrinology and University Of Manchester, Manchester, UK, David Clemmons, University of North Carolina, Chapel Hill, USA, Pinchas Cohen, UCLA Mattels Children's Hospital, Los Angeles, USA, Anna Maria Colao, University Federico II of Naples, Naples, Italy, Cheri Deal, University De Montreal, Montreal, Canada. Ezio Ghigo, University Of Turin, Turin, Italy, Naomi Hizuka, Japan Endocrine Society, Tokyo, Japan, Ken Ho, Garvan Institute of Medical Research, Sydney, Australia, Gudmundur Johannsson, Sahlgrenska Academy, Gothenburg, Sweden, Jens Otto Jorgensen, University of Aarhus, Aarhus, Denmark, Heike Jung,
Eli Lilly, Frankfurt, Germany. Anne-Marie Kappelgaard, Novo Nordisk, Denmark, John Kopchick, Ohio University, Athens, USA, Ione Kourides, Pfizer, New York, USA, Mohamad Maghnie, University of Genova, Genova, Italy, Saul Malozowski, National Institute of Health, Bethesda, USA, Nelly Mauras, Nemours Children's Clinic, Jacksonville, USA. Mark McLean, Endocrine Society of Australia, Sydney, Australia, Anne Nelson Garvan Institute of Medical Research, Sydney, Australia, Alan Rogol, Lawson Wilkins Paediatric Endocrine Society, Charlottesville, USA, Akira Sata, Garvan Institute Of Medical Research, Sydney, Australia, Christian Strasburger, Charite Universitatsmedizin, Berlin, Germany, Katsuhiko Tachibana, JCR Pharmaceuticals, Tokyo, Japan, Michael Thorner, University of Virginia, Charlottesville, USA, Susan Webb, Hospital de Sant Pau, Autonomous University of Barcelona, Barcelona, Spain.

\section{References}

1 Consensus Statement. Consensus guidelines for the diagnosis and treatment of adults with growth hormone deficiency: summary statement of the Growth Hormone Research Society Workshop on adult growth hormone deficiency. Journal of Clinical Endocrinology and Metabolism 199883 379-381.

2 Ghigo E, Aimaretti G \& Cornelli G. The diagnosis of adult growth hormone deficiency. Growth Hormone and IGF Research 2007.

3 Nilsson A, Svensson J \& Johannsson G. The management of growth hormone deficiency in adults. Growth Hormone and IGF Research 2007.

4 Clayton PE, Cuneo RC, Juul A, Monson JP, Shalet SM \& Tauber M. Consensus statement on the management of the GH-treated adolescent in the transition to adult care. European Journal of Endocrinology 2005152 165-170.

Received 16 September 2007

Accepted 1 October 2007 